Bull. Austral. Math. Soc.

20G35, 15A23

VOL. $61(2000) \quad[97-107]$

\title{
DECOMPOSITION OF THE STEINBERG GROUP OVER LOCAL RINGS INTO INVOLUTIONS
}

\author{
Ji ZhU NAN
}

We consider the stable Steinberg group $S t(R)$ over local rings. An element $x$ is called an involution if $x^{2}=1$. We prove that every element $\delta$ in $S t(R)$ is the product of at most 5 involutions.

\section{INTRODUCTION}

It is a classical problem in the research of classical groups to represent an element of a matrix group as a product of a special nature (such as of involutions and commutators) and to determine the smallest number of the factors in the representation $[1,2,3,4]$. It is known that every element of $S L_{n}(F)\left(=E_{n}(F)\right)$, the special linear group over a field, can be written as a product of at most four involutions for $n \geqslant 3$ [5]. The present note will consider the factorisation of stable Steinberg groups over local rings into involutions. Now let us introduce some definitions and propositions that will be used in our note $[6,7]$.

Definition: An element $x$ of a group is called an involution if $x^{2}=1$.

The Steinberg group $S t_{n}(R)(n \geqslant 3)$ over an associative ring (with 1$) R$ is the group with generators $x_{i j}(r)(r \in R, 1 \leqslant i, j \leqslant n)$, and relations:

(1) $x_{i j}(r) \cdot x_{i j}(s)=x_{i j}(r+s),(r, s \in R)$;

(2) $\left[x_{i j}(r), x_{k l}(s)\right] \begin{cases}x_{i l}(r s), & j=k, \\ 1, & j \neq k, i \neq l .\end{cases}$

Let $\varphi_{n}: S t_{n}(R) \rightarrow E_{n}(R)$ (the elementary linear group) be the natural epimorphism mapping $x_{i j}(r)$ to $e_{i j}(r)$. Denote $K_{2, n}(R)=\operatorname{ker} \varphi_{n}$. By passing to the direct limit as $n \rightarrow \infty$, we obtain the stable Steinberg group $S t(R)$ and the epimorphism $\varphi: S t(R) \rightarrow E(R)$. Denote $K_{2}(R)=\operatorname{ker} \varphi$. When $m \geqslant n$, define $f_{n, m}: S t_{n}(R) \rightarrow$ $S t_{m}(R)$ as the injective homomorphism. So $f_{n}: S t_{n}(R) \rightarrow S t(R)$ is the injection of $S t_{n}(R)$ into $S t(R)$. It is clear that $f_{n}=f_{m} \cdot f_{n, m}, f_{m}\left(K_{2, m}(R)\right) \supseteq f_{n}\left(K_{2, n}(R)\right)$, and $K_{2}(R)=\bigcup_{n \geqslant 3} K_{2, n}(R)$.

For any $u \in R^{*}$ (the set of units in $R$ ), define $w_{i j}(u)=x_{i j}(u) x_{j i}\left(-u^{-1}\right) x_{i j}(u)$, $h_{i j}(u)=w_{i j}(u) w_{i j}(-1)$.

Received 13th May, 1999

Copyright Clearance Centre, Inc. Serial-fee code: 0004-9727/00 \$A2.00+0.00. 
Proposition 1.1. $[6,7]$ Let $w \in S t_{n}(R), \varphi_{n}(w)=P(\pi) \operatorname{diag}\left(v_{1}, \ldots, v_{n}\right)$. If $\pi(i)=k$ and $\pi(j)=1$. We have

(1) $w x_{i j}(r) w^{-1}=x_{k l}\left(v_{i} r v_{j}^{-1}\right)(r \in R)$,

(2) $w w_{i j}(u) w^{-1}=w_{k l}\left(v_{i} u v_{j}^{-1}\right)\left(u \in R^{*}\right)$,

(3) $\quad w h_{i j}(u) w^{-1}=h_{k l}\left(v_{i} u v_{j}^{-1}\right) h_{k l}\left(v_{i} v_{j}^{-1}\right)^{-1}$.

Proposition 1.2. $[6,7]$ Let $u, v \in R^{*}$. We have

(1) $w_{i j}(u)=w_{j i}\left(-u^{-1}\right)$,

(2) $h_{i j}(u) h_{j i}(u)=1, h_{i j}(1)=1$,

(3) $\left[h_{i j}(u), h_{j k}(v)\right]=h_{i k}(u v) h_{i k}(u)^{-1} h_{i k}(v)^{-1}$.

DEFINITION: [8] $G L(R)=\bigcup_{n \geqslant 1} G L_{n}(R), E L(R)=\bigcup_{n \geqslant 1} E L_{n}(R)$. For any element $A$ in $G L_{n}(R)$, we can define an injective homomorphism $G L_{n}(R) \rightarrow G L_{m}(R)$ by

$$
\tau_{n, m}(A)=\left(\begin{array}{cc}
A & 0 \\
0 & I_{n-m}
\end{array}\right), \text { where } m \geqslant n
$$

For $m \geqslant n$, define an injective homomorphism by

$$
\begin{gathered}
f_{n, m}: S t_{n}(R) \rightarrow S t_{m}(R) \\
f_{n, m}\left(x_{i j}(a)\right)=x_{i j}(a) .
\end{gathered}
$$

Then $f_{n}=f_{m} \cdot f_{n, m}$, and we have the commutative diagram

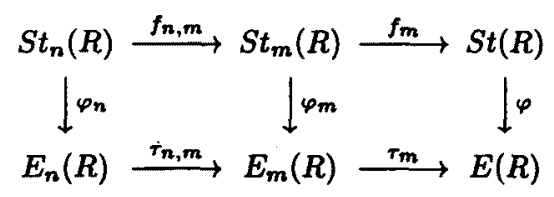

where $\tau_{n, m}(A)=\left(\begin{array}{cc}A & O \\ O & I_{n-m}\end{array}\right), \tau_{n}=\tau_{m} \cdot \tau_{n, m}$. It is clear that $S t_{m}(R) \supseteq S t_{n}(R)$ as subgroups of $S t(R)$ and that $S t(R)=\bigcup_{n \geqslant 3} S T_{n}(R)$. It follows from the above commutative diagram that for $m \geqslant n, K_{2, m}(R) \supseteq K_{2, n}(R)$ as subgroups of $K_{2}(R)$. Analogous to the situation above, we have $K_{2}(R)=\bigcup_{n \geqslant 3} K_{2, n}(R)$. If $R$ is a field, then $K_{2}(F) \cong K_{2, n}(F)$. Now let $R$ be a local ring. For any $u, v \neq 0 \in R$, define $\{u, v\}=h_{i k}(u v) h_{i k}(v)^{-1} h_{i k}(v)^{-1}$. By [7] we know that $K_{2}(R)$ is generated by the symbols $\{u, v\}$ and the symbols $\{u, v\}$ are independent of the choice of indices $i, k$. For the symbols $\{u, v\}$, we have

(1) $\{u, v\}^{-1}=\{v, u\}$,

(2) $\{u, 1-u\}=\{u,-u\}=1 \quad(u \neq 1)$,

(3) $\left\{u_{1} u_{2}, v\right\}=\left\{u_{1}, v\right\}\left\{u_{2}, v\right\},\left\{u, v_{1} v_{2}\right\}=\left\{u, v_{1}\right\}\left\{u, v_{2}\right\}$. 


\section{DEcomposition of Matrices OVER local RINGS}

Let $R$ denote a commutative local ring with maximal ideal $M, R / M$ the residue field and $R^{*}=R \backslash M$. As usual, $M_{n}(R)$ denotes the set of $n \times n$ matrices over $R$. By "-." we denote the natural ring morphism $R \rightarrow R / M$ and $M(R) \rightarrow M(R)$. Then it is easy to prove that $A \in G L_{n}(R)$ if and only if $\bar{A}=\left(\bar{a}_{i j}\right) \in G L_{n}(\bar{R})$.

In this section, we shall prove that every element $\delta$ in $S L_{n}(R)$ is the product of at most 5 involutions.

LEMma 2.1. [9] Every element of $S_{n}$, the group of permutations on $n$ letters, is the product of at most 2 involutions.

LemMa 2.2. Let $A$ be a matrix of the form $\left(\begin{array}{ccccc}* & * & \cdots & * & -b_{0} \\ 1 & * & \cdots & * & -b_{1} \\ * & 1 & \cdots & * & -b_{2} \\ \vdots & \vdots & \ddots & \vdots & \vdots \\ * & * & \cdots & 1 & -b_{n-1}\end{array}\right)$, where

$* \in M$. Then $A$ is similar to a matrix $\left(\begin{array}{ccccc}0 & 0 & \ldots & 0 & -a_{0} \\ 1 & 0 & \ldots & 0 & -a_{1} \\ 0 & 1 & \ldots & 0 & -a_{2} \\ \vdots & \vdots & \ddots & \vdots & \vdots \\ 0 & 0 & \ldots & 1 & -a_{n-1}\end{array}\right)$.

Proof: Without loss of generality, we prove it for $n=3$. Let $A=\left(\begin{array}{ccc}* & * & a_{0} \\ 1 & * & a_{1} \\ * & 1 & a_{2}\end{array}\right)$. Conjugating $A$ by $P_{1}=\left(\begin{array}{ccc}1 & * & \\ & 1 & \\ & & 1\end{array}\right)$, we have $P_{1} A P_{1}^{-1}=\left(\begin{array}{ccc}0 & * & a_{0} \\ 1 & * & a_{1} \\ * & 1+^{*} & a_{2}\end{array}\right)$. Now let $P_{2}\left(\begin{array}{ccc}1 & & \\ & 1 & \\ & * & 1\end{array}\right)$, then $P_{2} P_{1} A P_{1}^{-1} P_{1}^{-1}=\left(\begin{array}{ccc}0 & * & a_{0} \\ 1 & * & a_{1} \\ 0 & 1+* & a_{2}\end{array}\right)$. Further, if we let $P_{3}\left(\begin{array}{lll}1 & & * \\ & 1 & * \\ & & 1\end{array}\right)$, then $P_{3} P_{2} P_{1} A P_{1}^{-1} P_{2}^{-1} P_{3}^{-1}=\left(\begin{array}{ccc}0 & 0 & a_{0} \\ 1 & 0 & a_{1} \\ 0 & 1+^{*} & a_{2}\end{array}\right)$. Last, we may assume that $P_{4}=\left(\begin{array}{ccc}1 & & \\ & 1 & \\ & & \left(1++^{*}\right)^{-1}\end{array}\right)$, then we have $P_{4} P_{3} P_{2} P_{1} A P_{1}^{-1} P_{2}^{-1} P_{3}^{-1} P_{4}^{-1}=$ $\left(\begin{array}{lll}0 & 0 & a_{0} \\ 1 & 0 & a_{1} \\ 0 & 1 & a_{2}\end{array}\right)$. 
Lemma 2.3. Assume that $A \in S L_{n+1}(R), A=\left(\begin{array}{ll}B & \\ & 1\end{array}\right)$, where $B \in S L_{n}(R)$ and the characteristic polynomial of matrix $\bar{B}$ is irreducible. Then $A$ can be written as a product of at most 3 involutions and these involutions are in $S L_{n+1}(R)$.

ProOF: Without loss of generality, in the following discussion, we often write a matrix in its normal form of similarity. Now we may assume that

$$
\bar{B}=\left(\begin{array}{ccccc}
0 & 0 & \ldots & 0 & -a_{0} \\
1 & 0 & \ldots & 0 & -a_{1} \\
0 & 1 & \ldots & 0 & -a_{2} \\
\vdots & \vdots & \ddots & \vdots & \vdots \\
0 & 0 & \ldots & 1 & -a_{n-1}
\end{array}\right), \quad \text { so } B=\left(\begin{array}{ccccc}
* & * & \ldots & * & -b_{0} \\
1 & * & \ldots & * & -b_{1} \\
* & 1 & \ldots & * & -b_{2} \\
\vdots & \vdots & \ddots & \vdots & \vdots \\
* & * & \ldots & 1 & -b_{n-1}
\end{array}\right) \text {, }
$$

where the element ${ }^{*}$ is in the maximal ideal $M$. By Lemma 2.2, $B$ is similar to a matrix with the form

$$
\left(\begin{array}{ccccc}
0 & 0 & \ldots & 0 & -a_{0} \\
1 & 0 & \ldots & 0 & -a_{1} \\
0 & 1 & \ldots & 0 & -a_{2} \\
\vdots & \vdots & \ddots & \vdots & \vdots \\
0 & 0 & \ldots & 1 & -a_{n-1}
\end{array}\right)
$$

Thus we have $d_{1}, \ldots, d_{n-1} \in R$ such that

$$
\left(\begin{array}{cccc}
-1 & 0 & \ldots & 0 \\
d_{1} & 1 & \ldots & 0 \\
\vdots & \vdots & \ddots & \vdots \\
d_{n-1} & 0 & \ldots & 1
\end{array}\right)\left(\begin{array}{ccccc}
0 & 0 & \ldots & 0 & -a_{0} \\
1 & 0 & \ldots & 0 & -a_{1} \\
0 & 1 & \ldots & 0 & -a_{2} \\
\vdots & \vdots & \ddots & \vdots & \vdots \\
0 & 0 & \ldots & 1 & -a_{n-1}
\end{array}\right)=\left(\begin{array}{ccccc}
0 & 0 & \ldots & 0 & -a_{0} \\
1 & 0 & \ldots & 0 & 0 \\
0 & 1 & \ldots & 0 & 0 \\
\vdots & \vdots & \ddots & \vdots & \vdots \\
0 & 0 & \ldots & 1 & 0
\end{array}\right) .
$$

But

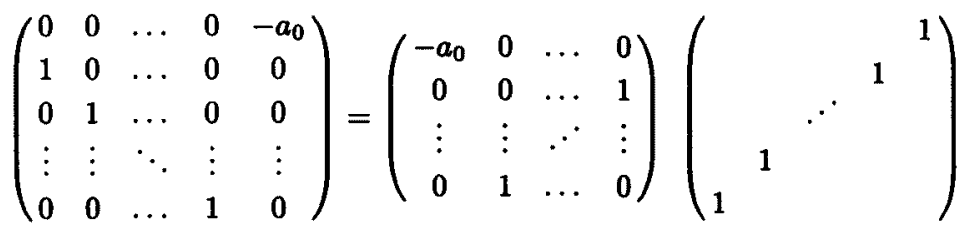

Hence $B$ is the product of at most 3 involutions. Since $B \in S L_{n}(R)$, we know that the number of involutions with determinant -1 is even, in the representation $B=H_{1} H_{2} H_{3}$ ( $H_{i}$ are involutions). Otherwise, we obtain $B \notin S L_{n}(R)$. Thus

$$
\left(\begin{array}{ll}
B & \\
& 1
\end{array}\right)=\left(\begin{array}{ll}
H_{1} & \\
& \pm 1
\end{array}\right)\left(\begin{array}{ll}
H_{2} & \\
& \pm 1
\end{array}\right)\left(\begin{array}{ll}
H_{3} & \\
& \pm 1
\end{array}\right) \text {. }
$$


When $\operatorname{det} H_{i}=-1$, we choose $\left(\begin{array}{cc}H_{i} & \\ & \pm 1\end{array}\right)=\left(\begin{array}{cc}H_{i} & \\ & -1\end{array}\right)$ and when $\operatorname{det} H_{i}=1$, we assume that $\left(\begin{array}{cc}H_{i} & \\ & \pm 1\end{array}\right)=\left(\begin{array}{cc}H_{i} & \\ & 1\end{array}\right)$. That is to say, $\left(\begin{array}{cc}H_{i} & \\ & \pm 1\end{array}\right) \in S L_{n+1}(R)$. $\quad$. REMARK. Obviously, we can assume that the matrices which are used in the above two lemmas to conjugate $A$ are in $S L_{n+1} R$. For example, if $P=\left(\begin{array}{cc}t & \\ & I\end{array}\right) \in G L_{n}(R)$, then we can take $P=\left(\begin{array}{ccc}t & & \\ & I & \\ & & t^{-1}\end{array}\right) \in S L_{n+1}(R)$; if $P=\left(\begin{array}{ccc}0 & 1 & \\ 1 & 0 & \\ & & I\end{array}\right)$, then we can take $P=\left(\begin{array}{cccc}0 & 1 & & \\ 1 & 0 & & \\ & & I & \\ & & & -1\end{array}\right) \in S L_{n+1}(R) ;$ If $P$ is the other elementary matrix, then we can let $P=\left(\begin{array}{cc}P & \\ & 1\end{array}\right)$

TheOREM 2.4. Let $A \in S L_{n+1}(R)$. If $A$ has the form $\left(\begin{array}{ll}B & \\ & 1\end{array}\right)$, where $B \in$ $S L_{n}(R)$, then $A$ is the product of at most 5 involutions and these involutions are in $S L_{n+1}(R)$.

Proof: Without loss of generality, we can suppose that $\bar{B}$ has the form

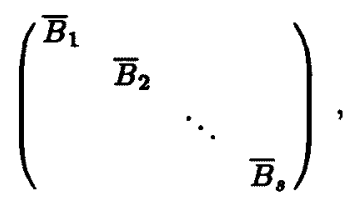

where $\bar{B}_{i}(1 \leq i \leq s)$ is a matrix with the form $\left(\begin{array}{ccccc}0 & 0 & \ldots & 0 & -a_{0} \\ 1 & 0 & \ldots & 0 & -a_{1} \\ 0 & 1 & \ldots & 0 & -a_{2} \\ \vdots & \vdots & \ddots & \vdots & \vdots \\ 0 & 0 & \ldots & 1 & -a_{m-1}\end{array}\right)$, or $\bar{B}_{i}$ is a diagonal matrix. Thus there is a matrix $P$ such that

$$
P B P^{-1}=\left(\begin{array}{cccc}
B_{1} & * & \ldots & * \\
* & B_{2} & \ddots & \vdots \\
\vdots & \ddots & \ddots & * \\
* & \ldots & * & B_{a}
\end{array}\right)
$$


where $B_{i}(1 \leqslant i \leqslant s)$ is equal to $\left(\begin{array}{ccccc}* & * & \ldots & * & -a_{0} \\ 1 & * & \ldots & * & -a_{1} \\ * & 1 & \ldots & * & -a_{2} \\ \vdots & \vdots & \ddots & \vdots & \vdots \\ * & * & \ldots & 1 & -a_{m-1}\end{array}\right)$ or $\left(\begin{array}{cccc}b_{1} & * & \ldots & * \\ * & b_{2} & \ddots & \vdots \\ \vdots & \ddots & \ddots & * \\ * & \ldots & * & b_{m}\end{array}\right)$.

Of course, the element ${ }^{*}$ is in $M$. Hence we have a permutation matrix $H$ such that

$$
H B=\left(\begin{array}{ccccc}
* & * & \cdots & * & a_{1} \\
a_{2} & * & & * & * \\
* & a_{3} & \ddots & \vdots & * \\
\vdots & \ddots & \ddots & * & \vdots \\
* & \ldots & * & a_{n} & *
\end{array}\right)
$$

where, these elements ${ }^{*}$ are in $M$. Then by Lemma 2.2 , matrix $H B$ is similar to a matrix

$$
\left(\begin{array}{ccccc}
0 & 0 & \ldots & 0 & -a_{0} \\
1 & 0 & \ldots & 0 & -a_{1} \\
0 & 1 & \ldots & 0 & -a_{2} \\
\vdots & \vdots & \ddots & \vdots & \vdots \\
0 & 0 & \ldots & 1 & -a_{m-1}
\end{array}\right)
$$

Now by Lemma 2.3 , matrix $H B$ can be written as a product of at most 3 involutions. On the other hand, $H$ is a product of at most 2 involutions by Lemma 2.1. Thus $B$ can be written as a product of at most 5 involutions.

Finally, using the same method as in Lemma 2.3 and Theorem 2.4, we can prove that those involutions which are in the representation of $B$ as above are in $S L_{n+1}(R)$.

\section{Decomposition of Steinberg Groups}

Since $\varphi: S t(R) \rightarrow E(R)$ is subjective, there is an element $\rho \in S T(R)$ such that $\varphi(\rho)=P$ for any given matrix $P$. Now we have $K_{2}(R)=\operatorname{ker} \varphi$ and it is the centre of the stable Steinberg group $S t(R)$ [7]. Thus for any $x \in S T(R)$, there exists $n \in \mathbf{Z}$ such that $\varphi(x) \in E_{n}(R)=S L_{n}(R)=\tau_{n+m, n}\left(S L_{n}(R)\right) \subseteq S L_{n+m}(R)$. Then by Theorem 2.4 , we have

$$
\varphi(x)=\mathrm{H}_{1} \mathrm{H}_{2} \mathrm{H}_{3} \mathrm{H}_{4} \mathrm{H}_{5}
$$

where $H_{i}$ is an involution in $S L_{n+m}(R)$, so of course, they are in $S L(R)=E(R)$. Hence if we find five involutions $\delta_{i}(1 \leqslant i \leqslant 5)$ in $S t(R)$ such that $\varphi\left(\delta_{i}\right)=H_{i}$, then we obtain

$$
x=\omega \cdot\left(\delta_{1} \delta_{2} \delta_{3} \delta_{4} \delta_{5}\right)
$$

where $\omega$ is in $\operatorname{ker} \varphi$ (the centre of $S t(R)$ ). 
We know that $H=\left(\begin{array}{ccc}0 & 1 & \\ 1 & 0 & \\ & & -1\end{array}\right)$ is an involution in $S L_{3}(R) \subseteq S L(R)$, but we easily obtain an element $w_{12}(1) h_{13}(-1) \in S t(R)$ such that $\varphi\left(w_{12}(1) h_{13}(-1)\right)=H$ and it is not an involution in $S t(R)[6]$. So we must show that for those involutions $H_{i}(1 \leqslant i \leqslant 5)$ in $S L(R)$ and $H_{1} H_{2} H_{3} H_{4} H_{5}$, we can find involutions $\delta_{i}(1 \leqslant i \leqslant 5)$ such that they are in $S t(R)$ and they satisfy $\varphi\left(\delta_{1} \delta_{2} \delta_{3} \delta_{4} \delta_{5}\right)=H_{1} H_{2} H_{3} H_{4} H_{5}$. On the other hand, if we prove that $\omega$ is a product of at most 5 involutions, of course, these involutions must be in $S t(R)$. If we prove that these involutions which occur in the representation of $\omega$ commute with $\delta_{i}$, then we obtain our main result.

Here we shall show that we can find involutions $\delta_{i}$ that satisfy the above conditions. By the proof of Theorem 2.4, we know that those involutions that occur in the representation of Theorem 2.4 occur in the decomposition of a permutation or in the decomposition of a matrix with the form $\left(\begin{array}{ccccc}0 & 0 & \ldots & 0 & -a_{0} \\ 1 & 0 & \ldots & 0 & -a_{1} \\ 0 & 1 & \ldots & 0 & -a_{2} \\ \vdots & \vdots & \ddots & \vdots & \vdots \\ 0 & 0 & \ldots & 1 & -a_{m-1}\end{array}\right)$. Now we consider the case of a permutation, written as the product of two involutions. In fact, a permutation $S$ with order $n$ can be written as a product of two involutions and these involutions are similar to the direct sum of involutions of the form $I_{1}=\left(\begin{array}{llll}0 & 1 & & \\ 1 & 0 & & \\ & 0 & 1 \\ & & 1 & 0\end{array}\right)$ and $I_{2}=\left(\begin{array}{lll}0 & 1 & \\ 1 & 0 & \\ & & -1\end{array}\right)$. Hence we only need show the simple case, that is to say, we can assume that

$$
S=P I_{1} P^{-1} \cdot Q I_{2} Q^{-1} \text {, or } S=P I_{1} P^{-1} \cdot Q I_{1} Q^{-1} \text { and } S=P I_{2} P^{-1} Q I_{2} Q^{-1} \text {. }
$$

But we can send $S L_{n}(R)$ to $S L_{m}(R)$ under $\tau_{n, m}$. So in $S L_{n+2}(R)$, we have

$$
\begin{aligned}
& S=\left(\begin{array}{cc}
P & \\
& I_{2 \times 2}
\end{array}\right)\left(\begin{array}{cc}
I_{1} & \\
& -I_{2 \times 2}
\end{array}\right)\left(\begin{array}{cc}
P^{-1} & \\
& I_{2 \times 2}
\end{array}\right) \\
& \cdot\left(\begin{array}{cc}
Q & \\
& I_{2 \times 2}
\end{array}\right)\left(\begin{array}{cc}
I_{2} & \\
& -I_{2 \times 2}
\end{array}\right)\left(\begin{array}{cc}
Q^{-1} & \\
& I_{2 \times 2}
\end{array}\right), \\
& \text { or } \quad \quad S=\left(\begin{array}{cc}
P & \\
& I_{2 \times 2}
\end{array}\right)\left(\begin{array}{cc}
I_{1} & \\
& -I_{2 \times 2}
\end{array}\right)\left(\begin{array}{cc}
P^{-1} & \\
& I_{2 \times 2}
\end{array}\right) \\
& \cdot\left(\begin{array}{cc}
Q & \\
& I_{2 \times 2}
\end{array}\right)\left(\begin{array}{cc}
I_{1} & \\
& -I_{2 \times 2}
\end{array}\right)\left(\begin{array}{cc}
Q^{-1} & \\
& I_{2 \times 2}
\end{array}\right),
\end{aligned}
$$


and

$$
\begin{aligned}
S=\left(\begin{array}{ll}
P & \\
& I_{2 \times 2}
\end{array}\right) & \left(\begin{array}{ll}
I_{2} & \\
& -I_{2 \times 2}
\end{array}\right)\left(\begin{array}{ll}
P^{-1} & \\
& I_{2 \times 2}
\end{array}\right) \\
& \cdot\left(\begin{array}{ll}
Q & \\
& I_{2 \times 2}
\end{array}\right)\left(\begin{array}{cc}
I_{2} & \\
& -I_{2 \times 2}
\end{array}\right)\left(\begin{array}{ll}
Q^{-1} & \\
& I_{2 \times 2}
\end{array}\right) .
\end{aligned}
$$

But by Propositon 1.1 and Proposition 1.2, we have

$$
\begin{aligned}
\varphi\left(w_{12}(1) h_{14}(-1) w_{34}(1) h_{56}(-1)\right) & =\left(\begin{array}{ll}
I_{1} & \\
& -I_{2 \times 2}
\end{array}\right), \\
\varphi\left(w_{12}(1) H_{13}(-1) h_{45}(-1)\right) & =\left(\begin{array}{ll}
I_{2} & \\
& -I_{2 \times 2}
\end{array}\right),
\end{aligned}
$$

where $w_{12}(1) h_{14}(-1) w_{34}(1) h_{56}(-1)$ and $w_{12}(1) h_{13}(-1) h_{45}(-1)$ are involutions in $S t(R)$.

$$
\text { Next, we consider the case } A=\left(\begin{array}{ccccc}
0 & 0 & \ldots & 0 & -a_{0} \\
1 & 0 & \ldots & 0 & -a_{1} \\
0 & 1 & \ldots & 0 & -a_{2} \\
\vdots & \vdots & \ddots & \vdots & \vdots \\
0 & 0 & \ldots & 1 & -a_{m-1}
\end{array}\right) \text { as a product of three }
$$

involutions. As in the proof of Theorem 2.4, we can assume

$$
\begin{aligned}
& A=\left(\begin{array}{cccc}
-1 & 0 & \ldots & 0 \\
d_{1} & 1 & \ldots & 0 \\
\vdots & \vdots & \ddots & \vdots \\
d_{n-1} & 0 & \ldots & 1
\end{array}\right)\left(\begin{array}{cccc}
-a_{0} & 0 & \ldots & 0 \\
0 & 0 & \ldots & 1 \\
\vdots & \vdots & . \cdot & \vdots \\
0 & 1 & \ldots & 0
\end{array}\right)\left(\begin{array}{ccc} 
& \\
& & \\
1 & & \\
1 & &
\end{array}\right)=X_{1} X_{2} X_{3} \\
& =\left(\begin{array}{cc}
-I_{3 \times 3} & \\
& X_{1}
\end{array}\right)\left(\begin{array}{cc}
-I_{3 \times 3} & \\
& X_{2}
\end{array}\right)\left(\begin{array}{cc}
-I_{3 \times 3} & \\
& X_{3}
\end{array}\right) \text {. }
\end{aligned}
$$

Thus we can use the same method, analogous to the situation above, to find two involutions $\delta_{1}$ and $\delta_{2}$ in $S t(R)$ such that $\varphi\left(\delta_{1} \delta_{2}\right)=\left(\begin{array}{ll}-I_{3 \times 3} & \\ & X_{2}\end{array}\right)\left(\begin{array}{cc}-I_{3 \times 3} & \\ & X_{3}\end{array}\right)$. Hence we only need show that we can also find an involution $\delta$ in $S t(R)$ such that $\varphi(\delta)=\left(\begin{array}{ll}-I_{3 \times 3} & \\ & X_{1}\end{array}\right)$. Without loss of generality, we let $\left(\begin{array}{cc}-I_{3 \times 3} & \\ & X_{1}\end{array}\right)=$

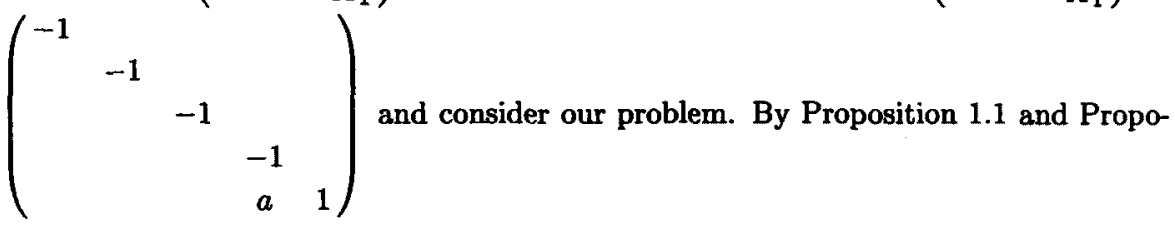


sition 1.2 , we have

$$
\varphi\left(h_{12}(-1) h_{34}(-1) x_{45}(a)\right)=\left(\begin{array}{ccccc}
-1 & & & & \\
& -1 & & & \\
& & -1 & & \\
& & & -1 & \\
& & & a & 1
\end{array}\right),
$$

where $h_{12}(-1) h_{34}(-1) x_{45}(a)$ is an involution in $S t(R)$.

So far, we have shown that there are involutions $\delta_{i}(1 \leqslant i \leqslant 5)$ in $\operatorname{St}(R)$ such that $\varphi\left(\delta_{1} \delta_{2} \delta_{3} \delta_{4} \delta_{5}\right)=H_{1} H_{2} H_{3} H_{4} H_{5}$.

Now we want to prove that $\omega$ is a product of at most 5 involutions; of course, these involutions must be in $S t(R)$. At the same time, we shall prove that these involutions occurring in the representation of $\omega$ commute with $\delta_{i}$. In order to complete the proof of the main result, let us prove the following lemma.

LEMMA 3.1. Let $R$ be a local ring. Then every element of $K_{2}(R)$ can be written as a product of at most four involutions.

Proof: 1. At first, let us consider the spacial case, the generator $\{u, v\}$. By definition

$$
\begin{aligned}
\{u, v\}= & h_{12}(u v) h_{12}(u)^{-1} h_{12}(v)^{-1}=h_{12}(u v) h_{21}(u) h_{21}(v) \\
= & w_{12}(u v) h_{13}(-1) h_{45}(-1) h_{54}(-1) h_{31}(-1) w_{12}(-1) . \\
& \quad w_{21}(u) h_{31}(-1) h_{45}(-1) h_{54}(-1) h_{13}(-1) w_{21}(-1) w_{21}(v) w_{21}(-1) .
\end{aligned}
$$

Since $h_{13}(-1) w_{12}(u) h_{13}(-1)^{-1}=w_{12}(-u)$, we have

$$
\begin{aligned}
\left(w_{12}(u) h_{13}(-1) h_{45}(-1)\right)^{2} & =w_{12}(u) w_{12}(-u)\left(h_{13}(-1)\right)^{2}\left(h_{45}(-1)\right)^{2} \\
& =\{-1,-1\}\{-1,-1\}=1 .
\end{aligned}
$$

That is, $w_{12}(u) h_{13}(-1) h_{45}(-1)$ is an involution in $S t(R)$. Similarly, $h_{54}(-1) h_{31}(-1) w_{12}(-1)$ and $h_{54}(-1) h_{13}(-1) w_{21}(-1) w_{21}(v) w_{21}(-1)$ are involutions in $\operatorname{St}(R)$.

2. General case. Every element $\omega$ of $K_{2}(R)$ can be written as $\omega \prod_{i=1}^{h}\left\{u_{i}, v_{i}\right\}$. Since the definition of $\left\{u_{i}, v_{i}\right\}$ is independent of the indices of $h_{k l}$, we can write $\left\{u_{i}, v_{i}\right\}=$ $T_{i}^{(1)} T_{i}^{(2)} T_{i}^{(3)} t_{i}^{(4)}$, where

$$
\begin{array}{r}
T_{i}^{(1)}=w_{5(i-1)+1,5(i-1)+2}\left(u_{i}, v_{i}\right) h_{5(i-1)+1,5(i-1)+3}(-1) h_{5(i-1)+4,5(i-1)+5}(-1) \\
T_{i}^{(2)}=h_{5(i-1)+5,5(i-1)+4}(-1) h_{5(i-1)+3,5(i-1)+1}(-1) w_{5(i-1)+1,5(i-1)+2}(-1) \\
T_{i}^{(3)}=w_{5(i-1)+2,5(i-1)+1}\left(u_{i}\right) h_{5(i-1)+3,5(i-1)+1}(-1) h_{5(i-1)+4,5(i-1)+5}(-1) \\
T_{i}^{(4)}=h_{5(i-1)+5,5(i-1)+4}(-1) h_{5(i-1)+1,5(i-1)+3}(-1) w_{5(i-1)+2,5(i-1)+1}(-1) \\
w_{5(i-1)+2,5(i-1)+1}\left(v_{i}\right) w_{5(i-1)+2,5(i-1)+1}(-1)
\end{array}
$$


are all involutions in $S t(R)$. Note that when $j \neq i$, the involutary factors in the factorisation of $\left\{u_{j}, v_{j}\right\}$ and $\left\{u_{i}, v_{i}\right\}$ are respectively exchangeable. So $\omega$ is a product of 4 involutions.

THEOREM 3.2. Let $R$ be a local ring, then every element of $S t(R)$ can be written as a product of at most 5 involutions.

Proof: We assume that $\xi \in S t(R)$. If $\xi \in K_{2}(R)$, then the conclusion of the theorem can be obtained by Lemma 3.1. Now suppose that $\xi \notin K_{2}(R)$. Then by the definition of $S t(R)$ there are a positive integer $n \geqslant 4$ and 5 involutions $H_{1}, H_{2}, H_{3}, H_{4}, H_{5} \in E_{n}(R)=S L_{n}(R)$ such that there are five involutions $\delta_{1}, \delta_{2}, \delta_{3}, \delta_{4}, \delta_{5} \in S t_{n}(R)$ such that $H_{i}=\varphi\left(\delta_{i}\right), \varphi(\xi)=H_{1} H_{2} H_{3} H_{4} H_{5}$. Thus we have

$$
\varphi(\xi)=\varphi\left(\delta_{1} \delta_{2} \delta_{3} \delta_{4} \delta_{5}\right), \text { that is }, \xi=\omega . \delta_{1} \delta_{2} \delta_{3} \delta_{4} \delta_{5}
$$

where $\omega \in K_{2}(R)$.

Let $\omega=\prod_{i=1}^{t}\left\{a_{i}, b_{i}\right\}$. Since the symbol $\left\{a_{i}, b_{i}\right\}$ is independent of the index of $h_{r k}$ occurring in the resresentation of $\left\{a_{i}, b_{i}\right\}$, we can choose sufficient large $r, k$ (all larger than $2 n$ ) such that

$$
\begin{aligned}
\left\{a_{i}, b_{i}\right\}=h_{2 n+5(i-1)+1,2 n+5(i-1)+2}\left(a_{i} b_{i}\right) h_{2 n+5(i-1)+1,2 n+5(i-1)+2}\left(a_{i}\right)^{-1} & \\
& h_{2 n+5(i-1)+1,2 n+5(i-1)+2}\left(b_{i}\right)^{-1} .
\end{aligned}
$$

By Lemma 3.1, $\omega$ is a product of 4 involutions $T_{1}, T_{2}, T_{3}, T_{4}$, but the indices $r, k$ of $h_{r k}, \omega_{r k}$ occurring in the representations of $T_{i}$ are larger than $2 n$. Thus $T_{i}$ commutes with $\delta_{i}$. So we have

$$
\begin{aligned}
\xi & =\left(T_{1} T_{2} T_{3} T_{4}\right)\left(\delta_{1} \delta_{2} \delta_{3} \delta_{4} \delta_{5}\right) \\
& =\left(T_{1} \delta_{1}\right)\left(T_{2} \delta_{2}\right)\left(T_{3} \delta_{3}\right)\left(T_{4}, \delta_{4}\right) \delta_{5}
\end{aligned}
$$

is a product of five involutions, and also we have that these involutions are in $S t(R) .0$

\section{REFERENCES}

[1] E. Ambrosiewicz, 'Powers of set of involutions in linear group', Demonstratio. Math 24 (1991), 311-314.

[2] R.K. Dennis and L.N. Vaserstein, 'On a question of M. Newman on the number of commutators', J. Algebra 118 (1988), 150-161.

[3] W.H. Gustafson, 'On products of involutions', in Paul Halmos celebrating 50 years of Mathematics (Springer-Verlag, Berlin, Heidelberg, New York, 1991). 
[4] W.H. Gustafson, P.R. Halmos and H. Radjavi, 'Products of involutions', Linear Algebra Appl. 13 (1976), 157-162.

[5] A. Hahn and O.T. O'Meara, The classical groups and $K$-theory (Springer-Verlang, Berlin, Heidelberg, New York, 1989).

[6] F. Knuppel and K. Nielsen, ' $S L(V)$ is 4-involutional', Geom. Dedicata 38 (1991), 301-308.

[7] J. Milnor, Introduction to algebraic $K$-theory (Princeton University Press, Princeton, 1971).

[8] J.R. Silvester, Introduction to algebraic $K$-theory (Chapman and Hall, London, New York, 1981).

[9] H. You and J.Z. Nan, 'Decomposition of matrices into 2-involutions', Linear Algebra Appl. 186 (1993), 235-243.

Department of Mathematics

Northeast Normal University

Chang Chun 130024

China 